\title{
SYNTHESIS OF 2-(1-(4-CHLOROPHENYL) ETHYL)-1-(2- (DIETHYLAMINO) ETHYL)-1H-BENZO[D]IMIDAZOL-5- AMINE
}

\author{
Siddhartha Marupati ${ }^{1,2}$, Laxminarayana Eppakayala ${ }^{3}$ and Gade Dayakar $^{1^{*}}$ \\ ${ }^{1}$ Departmet of Chemistry, Kakatiya University, Warangal-506009, Telangana, India \\ ${ }^{2}$ Vardhaman College of Engineering, Shamshabad, Hyderabad-500 018, Telangana, India \\ ${ }^{3}$ Sreenidhi Institute of Science and Technology (Autonomous) Yamnampet, Ghatkesar, \\ Hyderabad-501301, Telangana, India \\ *E-mail : gadedayakar@yahoo.co.in.
}

\begin{abstract}
A novel 2-(1-(4-chlorophenyl) ethyl)-1-(2-(diethylamino) ethyl)-1H-benzo[d]imidazol-5-amine is synthesized. The IR, NMR spectroscopy and mass spectrometry were used for the assessment of the structure of the synthesized compounds.
\end{abstract}

Keywords: Benzimidazole, $\mathrm{N}^{1}, \mathrm{~N}^{1}$-diethylethane-1, 2-diamine, Spectral analysis

(C) RASĀYAN. All rights reserved

\section{INTRODUCTION}

Benzimidazole derivatives are usually connected with different types of pharmacokinetic and pharmacodynamic properties. Benzimidazole derivatives possess pharmacological and biological activities $^{1}$, including antimicrobial ${ }^{2}$, diuretic ${ }^{3}$, antidiabetic $^{4}$, analgesic $^{5}$, antivitral ${ }^{6}$, antitumor ${ }^{7}$, antiulcer ${ }^{8}$, antioxidant $^{9}$, anthelmintic ${ }^{10}$, anti inflammatory ${ }^{11}$ and cysticidal activities ${ }^{12}$. Benzofuran is highly promising pharmacophore for the discovery of drugs, and numerical synthetic methodologies for benzofuran derivatives have been developed over the years. Specifically, vitamin B $12^{10}$ constituent of Benzofuran nucleus. The biological significance of the benzimidazole containing moiety is documented ${ }^{13}$, ${ }^{14}$ well. Albendazole, Mebendazole and Thiabendazole are commonly used as anthelmintic drugs ${ }^{15}$.

Benzimidazole derivatives show significance biological activities. Some of the already synthesized compounds from field mentioned above have been to have very strong applications in the field of medicine ${ }^{16}$. The antimicrobial activities resulted in their mode of action, which in turn resulted in the blockage of microtubule in the various nematode, trematode and cystode.

It was obvious from the literature described above that very little work seems to have been done on the reactions of orthophenylenediamine with aromatic acids and their further chemical modifications. Hence, it is thought appropriate to study the condensation of $o$-phenylenediamine analogs with carboxylic acids and further modified the cyclization products to achieve diversity-oriented synthesis. In continuous to our earlier work $^{17}$, we report synthesis of 2-(1-(4-chlorophenyl) ethyl)-1-(2-(diethylamino) ethyl)-1Hbenzo[d]imidazol-5-amine and 2-(2-(1-(4-chlorophenyl) ethyl)-5-nitro-1H-benzo[d]imidazol-1-yl)-N, Ndiethylethanamine as new chemical entities.

\section{EXPERIMENTAL}

$\mathbf{N}^{1}$-(2,4-dinitrophenyl)-N2,N2-diethylethane-1,2-diamine (3)

To a stirred solution of 1-chloro-2,4-dinitrobenzene $(15 \mathrm{~g}, 74.05 \mathrm{mmol})$ in $\mathrm{EtOH}(150 \mathrm{~mL})$ was added $\mathrm{N}^{1}, \mathrm{~N}^{1}$-diethylethane-1,2-diamine $(12.94 \mathrm{~g}, 111.08 \mathrm{mmol})$ at $\mathrm{RT}$, heated to 80 to $85{ }^{\circ} \mathrm{C}$ for $16 \mathrm{~h}$. Aq $\mathrm{NH}_{4}$ solution was added solid was formed which was filtered to afford $15 \mathrm{~g}(72 \%)$ of $\mathrm{N}^{1}$-(2,4-dinitrophenyl)$\mathrm{N}^{2}, \mathrm{~N}^{2}$-diethylethane-1,2-diamine (3) as solid.

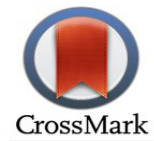


RASĀYAN J. Chem.

Vol. 11 | No. 4 |1674 - 1677| October - December | 2018

\section{TLC System}

EtOAc: pet ether (4: 6); $\mathrm{R}_{\mathrm{f}}: 0.2$

${ }^{1} \mathrm{H}$ NMR $\left(400 \mathrm{MHz}, \mathrm{CDCl}_{3}\right): 9.15(\mathrm{~d}, 1 \mathrm{H}, \mathrm{J}=2.8 \mathrm{~Hz}), 9.14$ (brs, $\left.1 \mathrm{H}\right), 8.26$ (dd, 1H, J= 9.6 \& $\left.2.4 \mathrm{~Hz}\right), 6.87$ $(\mathrm{d}, 1 \mathrm{H}, \mathrm{J}=9.6 \mathrm{~Hz}), 3.40(\mathrm{q}, 2 \mathrm{H}), 2.80(\mathrm{t}, 2 \mathrm{H}), 2.60(\mathrm{q}, 4 \mathrm{H}), 1.08(\mathrm{t}, 6 \mathrm{H})$;

Mass: $\left(\mathrm{m} / \mathrm{z}=283.1(\mathrm{M}+\mathrm{H})^{+}\right)$.

$\mathrm{N}^{1}$-(2-(diethylamino) ethyl)-4-nitrobenzene-1, 2-diamine (4)

$\mathrm{N}^{1}$-(2,4-dinitrophenyl)- $\mathrm{N}^{2}, \mathrm{~N}^{2}$-diethylethane-1,2-diamine (3) $(15 \mathrm{~g}, 53.19 \mathrm{mmol})$ was combined with EtOH $(150 \mathrm{~mL})$, which was subsequently added to a pre-mixed solution of aqueous; ammonium sulphide $(10.8 \mathrm{~g}, 159.57 \mathrm{mmol})$ in $\mathrm{EtOH}(300 \mathrm{~mL})$ and Water $(150 \mathrm{~mL})$, at $60{ }^{\circ} \mathrm{C}$ for $30 \mathrm{~min}$ and the reaction mixture was stirred for $24 \mathrm{~h}$ at $80-85^{\circ} \mathrm{C}$. Completion of the reaction was monitored by TLC. Water was added to the reaction mixture and extracted with DCM. Column chromatography was used for the purification of crude compound over silica gel (100 - 200 mesh) using a solvent gradient of $2-3 \%$ $\mathrm{MeOH}$ in $\mathrm{CHCl}_{3}$ as eluent to afford $11 \mathrm{~g}(80 \%)$ of $\mathrm{N}^{1}$-(2-(diethylamino)ethyl)-4-nitrobenzene-1,2diamine (4) as solid.

\section{TLC System}

$\mathrm{MeOH}: \mathrm{CHCl}_{3}(1: 9) ; \mathrm{R}_{\mathrm{f}}: 0.3$

${ }^{1} \mathrm{H}$ NMR $\left(400 \mathrm{MHz}, \mathrm{CDCl}_{3}\right): 7.81(\mathrm{dd}, 1 \mathrm{H}, \mathrm{J}=8.8 \mathrm{~Hz} \& 2.0 \mathrm{~Hz}), 7.60(\mathrm{~d}, 1 \mathrm{H}, \mathrm{J}=2.4 \mathrm{~Hz}), 6.51(\mathrm{~d}, 1 \mathrm{H}, \mathrm{J}=$ $8.8 \mathrm{~Hz}), 5.11$ (brs, 1H), 3.20 (q, 2H), $2.76(\mathrm{t}, 2 \mathrm{H}), 2.56$ (q, 4H), $1.04(\mathrm{t}, 6 \mathrm{H})$;

Mass: $\left(\mathrm{m} / \mathrm{z}=252(\mathrm{M}+\mathrm{H})^{+}\right)$.

\section{2-(4-chlorophenyl)-N-(2-(2-(diethylamino) ethylamino)-5-nitrophenyl) propanamide (6)}

$\mathrm{N}^{1}$-(2-(diethylamino)ethyl)-4-nitrobenzene-1,2-diamine (4) (11 g, $\left.43.65 \mathrm{mmol}\right)$ dissolved in DCM (150 $\mathrm{mL}$ ) was added 2-(4-Chloro-phenyl)-propionic acid (5) (11.24 g, $61.11 \mathrm{mmol})$, EEDQ (10.78 g, 43.65 $\mathrm{mmol}$ ) at RT heated and refluxed for $24 \mathrm{~h}$ at 50 to $55^{\circ} \mathrm{C}$. Completion of the reaction was monitored by TLC. To the reaction mixture $\mathrm{Aq} \mathrm{NH}_{4}$ solution was added and extracted with DCM. The column chromatography was used for the purification of crude compound over silica gel (100 - 200 mesh) using a solvent gradient of $1 \% \mathrm{MeOH}$ in DCM as eluent to afford $6 \mathrm{~g}$ (35\%) of 2-(4-chlorophenyl)-N-(2-(2(diethylamino)ethylamino)-5-nitrophenyl)propanamide (6) as solid.

${ }^{1} \mathrm{H}$ NMR (400 MHz, $\mathrm{CDCl}_{3}$ ): $8.04(\mathrm{~d}, 1 \mathrm{H}, \mathrm{J}=9.2 \mathrm{~Hz}), 8.01(\mathrm{~s}, 1 \mathrm{H}), 7.36$ (dd, 4H, J = 8.0 Hz), 6.63 (brs, $1 \mathrm{H}), 6.57(\mathrm{~d}, 1 \mathrm{H}, \mathrm{J}=8.8 \mathrm{~Hz}), 5.29(\mathrm{~s}, 1 \mathrm{H}), 3.74(\mathrm{q}, 1 \mathrm{H}), 3.15(\mathrm{q}, 2 \mathrm{H}), 2.67(\mathrm{~m}, 2 \mathrm{H}), 2.53(\mathrm{q}, 4 \mathrm{H}), 1.61(\mathrm{~d}$, $3 \mathrm{H}), 1.00$ (t, 6H);

Mass: $\left(\mathrm{m} / \mathrm{z}=419.1(\mathrm{M}+\mathrm{H})^{+}\right)$.

\section{2-(2-(1-(4-chlorophenyl) ethyl)-5-nitro-1H-benzo[d] midazole-1-yl)-N, N-diethylethanamine (7)}

To a stirred solution of 2-(4-chlorophenyl)-N-(2-(2-(diethylamino)ethylamino)-5nitrophenyl)propanamide (6) $(2.5 \mathrm{~g}, 5.98 \mathrm{mmol})$ in $\mathrm{CHCl}_{3}(50 \mathrm{~mL})$ was added $\mathrm{PCl}_{5}(4.9 \mathrm{~g}, 23.92 \mathrm{mmol})$ at RT heated and refluxed fro overnight . Completion of the reaction mixture was monitored by TLC. Aq $\mathrm{NH}_{4}$ solution was added to the reaction mixture at $0{ }^{\circ} \mathrm{C}$ and extracted with $\mathrm{CHCl}_{3}$. The column chromatography was used for the purification of crude compound over silica gel (100 - 200 mesh) using a solvent gradient of $1-1.5 \% \mathrm{MeOH}$ in $\mathrm{CHCl}_{3}$ as eluent to afford $2 \mathrm{~g}$ (83\%) of 2-(2-(1-(4-chlorophenyl) ethyl)-5-nitro-1H-benzo[d] midazole-1-yl)-N, N-diethylethanamine (7) as solid.

${ }^{1} \mathrm{H}$ NMR $\left(400 \mathrm{MHz}, \mathrm{CDCl}_{3}\right): 8.74(\mathrm{~d}, 1 \mathrm{H}, \mathrm{J}=2.4 \mathrm{~Hz}), 8.20(\mathrm{dd}, 1 \mathrm{H}, \mathrm{J}=8.8 \& 2.0 \mathrm{~Hz}), 7.33(\mathrm{~d}, 1 \mathrm{H}, \mathrm{J}=$ $9.2 \mathrm{~Hz}), 7.28(\mathrm{~d}, 2 \mathrm{H}, \mathrm{J}=8.4 \mathrm{~Hz}), 7.16(\mathrm{~d}, 2 \mathrm{H}, \mathrm{J}=8.4 \mathrm{~Hz}), 4.50(\mathrm{q}, 1 \mathrm{H}), 4.00(\mathrm{t}, 2 \mathrm{H}), 2.57(\mathrm{~m}, 1 \mathrm{H}), 2.51$ (m, 1H), $2.39(\mathrm{~m}, 4 \mathrm{H}), 1.83(\mathrm{~d}, 3 \mathrm{H}, \mathrm{j}=5.4 \mathrm{~Hz}), 0.86(\mathrm{t}, 6 \mathrm{H})$;

Mass: $\left(\mathrm{m} / \mathrm{z}=4.1 .2(\mathrm{M}+\mathrm{H})^{+}\right)$.

\section{2-(1-(4-chlorophenyl)ethyl)-1-(2-(diethylamino)ethyl)-1H-benzo[d]imidazole-5-amine (8)}

2-(2-(1-(4-chlorophenyl)ethyl)-5-nitro-1H-benzo[d]imidazole-1-yl)-N,N-diethylethanamine (7) (2.4 g, $6.0 \mathrm{mmol})$ was combined with $\mathrm{MeOH}(40 \mathrm{~mL})$ which was subsequently added to a prepared solution of saturated Aq; $\mathrm{NH}_{4} \mathrm{Cl}(20 \mathrm{~mL})$, Zinc powder $(4.0 \mathrm{~g}, 60.0 \mathrm{mmol})$ at $0{ }^{\circ} \mathrm{C}$ and stirred at $\mathrm{RT}$ for overnight. Sat $\mathrm{NaHCO}_{3}$ solution was added to the reaction mixture and extracted with EtOAc $(2 \times 100 \mathrm{~mL})$ The organic layer was concentrated under reduced pressure after it was washed with water, brine and dried over 
(anhyd. $\mathrm{Na}_{2} \mathrm{SO}_{4}$ ). The column chromatography was used for the purification of crude compound over silica gel (100 - 200 mesh) using a solvent gradient of $2-2.5 \% \mathrm{MeOH}$ in $\mathrm{CHCl}_{3}$ as eluent to afford $1.2 \mathrm{~g}$ of 2-(1-(4-chlorophenyl) ethyl)-1-(2-(diethylamino) ethyl)-1H-benzo[d]imidazole-5-amine (8) as solid.

\section{TLC System}

$\mathrm{MeOH}: \mathrm{CHCl}_{3}(1: 9) ; \mathrm{R}_{\mathrm{f}}: 0.3$

${ }^{1} \mathrm{H}$ NMR (400 MHz, $\left.\mathrm{CDCl}_{3}\right): 7.36(\mathrm{dd}, 4 \mathrm{H}, \mathrm{J}=8.2 \mathrm{~Hz}), 7.12(\mathrm{~d}, 1 \mathrm{H}, \mathrm{J}=8.8 \mathrm{~Hz}), 6.82(\mathrm{~s}, 1 \mathrm{H}), 6.61$ (dd, $1 \mathrm{H}, \mathrm{J}=8.2 \& 2.0 \mathrm{~Hz}) 4.52(\mathrm{q}, 1 \mathrm{H}), 4.01(\mathrm{~m}, 2 \mathrm{H}), 2.52-2.16(\mathrm{~m}, 6 \mathrm{H}), 1.62(\mathrm{~d}, 3 \mathrm{H}, \mathrm{J}=9.2 \mathrm{~Hz}), 0.78(\mathrm{t}$, $6 \mathrm{H})$;

Mass: $\left(\mathrm{m} / \mathrm{z}=370.9(\mathrm{M}+\mathrm{H})^{+}\right)$.<smiles>O=[N+]([O-])c1ccc(Cl)c([N+](=O)[O-])c1</smiles><smiles>CCOC(C)(CN(CC)CC)C(C)O</smiles><smiles>CC(=O)C(C)c1ccc(Cl)cc1</smiles>

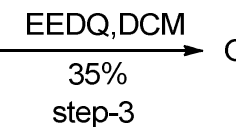<smiles>CCN(CC)CCNc1ccc([N+](=O)[O-])cc1[N+](=O)[O-]</smiles>

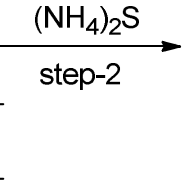<smiles>CCN(CC)CCNc1ccc([N+](=O)[O-])cc1N</smiles>

.


RASĀYAN J. Chem.

Vol. 11 | No. 4 |1674 - 1677| October - December | 2018

3. N. Srinivasan, A. Balaji, G. Nagarajan, R. Suthakaran, Y. Kumar, D. Jagadesh, Asian J. Chem., 20, 4934 (2008).

4. B. Kumar, P. Rao, Asian J. Chem., 18, 3060 (2006).

5. T. Solominova, V. Pilyugin, A. Tyurin, A. Kirlan, L. Tyurina, Pharm. Chem. J., 38, 425 (2004).

6. R. V. Devivar, E. Kawashima, G. R. Revankar, J. M. Breitenbach, E. D. Kreske, J. C. Drach, L. B. Townsend, J Med Chem., 37, 2942 (1994).

7. A. Gellis, H. Kovacic, N. Boufatah, P. Vanelle, Eur. J. Med. Chem, 43, 1858 (2008).

8. J. Bariwal, A. Shah, M. Kathiravan, R. Somani, J. Jagtap, K. Jain, Indian J Pharm Educ., 42, 225 (2008).

9. Z. Alagoz, C. Kus, T. Coban, J Enzyme Inhib MedChem., 20, 325 (2004).

10. A. Lee-Dutra, K. L. Arienti, D. J. Buzard, M. D. Hack, H. Khatuya, P. J. Desai, S. Nguyen, R. L. Thurmond, L. Karlsson, J. P. Edwards, Bioorganic Med. Chem. Lett, 16, 6043 (2006).

11. J. Leonard, L. Jeyaseeli, O. Rajesh, K. Murugesh, R. Sivakumar, V. Gunasekaran, Asian J. Chem., 18, 1104 (2006).

12. Palomares-Alonso, H. Jung-Cook, J. Pérez-Villanueva, J. C. Piliado, S. Rodríguez-Morales, G. Palencia-Hernández, N. López-Balbiaux, A. Hernández-Campos, R. Castillo, F. Hernández-Luis, Eur. J. Med. Chem, 44, 1794 (2009).

13. M. J. O’Neil, A. Smith, P. Heckelman, J. Obenchain, J. Gallipeau, M. D'arecca, Inc., Whitehouse Station, NJ, 309, 405 (2001).

14. M. Amari, M. Fodili, B. Nedjar Kolli, A. P. Hoffmann, J. Perie, J. Heterocycl. Chem, 39, 811 (2002).

15. P. Köhler, Int J Parasitol., 31, 336 (2001).

16. A. T. Mavrova, K. K. Anichina, D. I. Vuchev, J. A. Tsenov, P. S. Denkova, M. S. Kondeva, M. K. Micheva, Eur. J. Med. Chem, 41, 1412 (2006).

17. M. Siddhartha, E. Laxminarayana, N. Saroja, M. Ramesh M. Ramchander, Rasayan J. Chem, 10(4), 1094 (2017).

[RJC-4087/2018] 